\title{
Increasing uptake of bowel cancer screening
}

\author{
Sarah Graham
}

Prince of Wales Road Group Practice, England

\begin{abstract}
Uptake of bowel cancer screening uptake at our practice is $32.72 \%$, which is below the national target of $60 \%$, but our cancer prevalence and death rate is higher than our CCG statistical mean. We examined reasons for non-response to bowel cancer screening in our patients and explored ways to promote engagement.
\end{abstract}

From August 2013 to February 2014 we used three interventions in two patient groups: those turning 60 and eligible for screening (rising 60's) and non-responders to screening. Interventions used were; letter encouragement for rising 60's, staff education to increase opportunistic promotion of screening and calling non- responders to identify reasons for non-participation and encourage participation. Calls were made by either a Doctor or a Health Care Assistant (HCA); ethnicity, language spoken, caller and call outcome was recorded.

Rising 60's ( $n=26$ ) had an uptake of $46 \%$, increased from $32.72 \%$. From the non-responders $(n=73$ ) we were unable to contact $38 \%$, $46 \%$ was due to an incorrect or no phone number. Of those contacted main reasons for non-participation were not receiving a screening kit ( $n=19)$ and not wanting to be screened $(n=14)$. Following calls $66 \%$ of non-responders agreed to screening. From this $66 \%$ half $(50 \%)$ completed screening with a negative result. 15 non-responders refused screening following our calls, the main reason given was not wanting to know if they had cancer $(n=14)$. Calls from doctor and HCA had similar rates of screening uptake ( $39 \%$ and $33 \%$ respectively).

Difficulty contacting patients was an unexpected barrier to screening and will be addressed. Actively encouraging screening appears beneficial with similar responses to Doctor and HCA. There appears to be a place for increased education regarding screening and early detection of malignancy amongst patients.

Overall our interventions improved screening uptake at the practice and will be continued in future.

\section{Problem}

Our practice is located in Camden, London. Cancer is leading cause of premature (age under 75) death in Camden (1). Screening uptake of all national screening programmes is below national targets in Camden with bowel cancer screening uptake at $39.86 \%$ below the national target of $60 \%$ (1). Our practice has a cancer prevalence of $1.9 \%$, higher than the Camden average of $1.4 \%$ (2), and the practice cancer death rate is significantly different from the CCG statistical mean (3) however when measured in March 2013 we had the third lowest uptake of bowel cancer screening in Camden (1).

In addition we are among the top four most deprived practices in Camden (4) and have high ethnic diversity. A large cross sectional analysis of colorectal cancer screening uptake data found 'there was low uptake in the most ethnically diverse areas and a striking gradient by socio economic status' (5). As a practice we therefore have an increased need for screening but our patients are potentially less likely to engage in screening.

\section{Background}

Approximately 100 new cases of bowel cancer are diagnosed each day in the UK making it the third commonest cancer and second commonest cause of cancer death in the UK (1). 16000 people die from bowel cancer each year in the UK (6) and risk increases with age, with eight out of ten people diagnosed over the age of 60 (6).

Bowel cancer is staged as A-D according to the Dukes Criteria. Early detection (stage A) has a 5-year survival rate of $85-95 \%$ whereas late detection (stage $D$ ) has survival rates less than $10 \%$ (1). Since 2006 the UK has had a national bowel cancer screening programme; the Faecal Occult Blood Test (FOBT) $(1,6)$, which checks stool samples for the presence of microscopic blood. It is offered at two yearly intervals to everyone aged 60-74 (6) with the aim of detecting asymptomatic cancer at an early stage, $68 \%$ of cancers detected at screening are Dukes Stage B or less (1).

Screening using FOBT has been proved to be effective with RCT's showing a $16 \%$ reduction in bowel cancer mortality with annual screening $(1,7)$ and a $15 \%$ reduction with biennial screening $(1,7)$.

\section{Baseline measurement}

This project was conducted as part of the 'Bowel Cancer Screening in Camden' locally enhanced service (1), initiated to increase bowel cancer screening uptake in Camden. As part of the LES data of current screening uptake for all Camden GP practices' was provided. This showed our practice had an uptake in 2012 of $33.97 \%$ which fell in March 2013 to $32.72 \%$ (1). 


\section{Design}

Our project ran from August 2013 to February 2014. In designing our project we wanted to answer two questions:

1. Why aren't our patients engaging with FOBT screening?

2. How can we increase FOBT screening uptake?

We focused on two groups of patients: 'rising 60's' and 'nonresponders'. Rising 60's were patients turning 60 and therefore eligible for screening and non-responders were patients who had failed to respond to an invite to participate in the screening programme i.e. had not returned a completed screening kit. The practice had been involved in the LES for a few months prior to the project starting and had started to send reminder letters to rising 60 's but awareness of the project was low and it was felt further interventions were needed.

Our interventions were:

1. To continue to send personalised letters from the practice were sent to all eligible rising 60's in the month of their 60th birthday to encourage participation in screening. A second personalised reminder letter was also sent four weeks later, aimed to arrive after they had received the screening kit.

2. To increase awareness all clinical staff were informed of the problem and reasons for the interventions. They were informed of the project and given a 'bowel cancer pack' containing information and sample screening kits. Alerts were placed on the notes of non-responders to promote opportunistic discussion and promotion of screening and the bowel cancer screening contact number was uploaded onto all practice computer desktops.

3. Monthly calls were made to all non-responders to identify reasons for non-participation and to encourage participation. Calls (total $n=73$ ) were divided and made by a Doctor $(n=46)$ or a Health Care Assistant (HCA) $(n=27)$. This was to allow us to assess response rate depending on caller to help plan the future sustainability of the project. A pro-forma was used to record reason for non-participation and call outcome following discussion.

\section{Strategy}

PDSA cycle 1: Once we started our calls it became apparent that the main reason for non-participation was 'not wanting to be screened', we wanted to obtain more detail about this so we altered the pro-forma to include more details about reasons for nonparticipation and how we could promote participation. Also ethnicity did not appear to be as large a barrier as we had suspected, we therefore also added information about ethnicity and language spoken to the pro-forma and back entered details of patients already called to allow us to look at this further.

PDSA cycle 2: An unseen barrier that emerged once we started our calls was that we often had incorrect contact details for patients. Our process was therefore adjusted so that if the phone contact details were incorrect a letter was sent to the patient and alerts were placed on the notes to highlight the need to confirm correct details to staff.

PDSA cycle 3: We collated our results to our calls at three months and, whilst we appeared to be getting a good response rate to participate on calls, this was not being reflected in kits returned. We therefore decided to make a follow up 'encouragement call' to each person who agreed to participate but had not returned a screening kit. This was done retrospectively for those who had agreed from August to December and two to four weekly after agreeing to participate from December onwards.

PDSA cycle 4: At three months we also discussed the project informally with the doctors in the practice who fed back that they would like more updates about the project and its progress. We therefore started to send 'update' emails giving information on progress and feedback from patients. These were sent at two monthly intervals.

PDSA cycle 5: At the project end as a main reason given for non participation was 'not wanting to be screened' to assess other ways to encourage participation we decided to call patients who had taken part in screening to assess what had made them participate. We randomly selected and called ten patients who had participated to discuss this with them. Unfortunately this did not prove overly helpful, as the main response we got was 'it just seemed sensible' or 'why would you not take part in screening?'

\section{Results}

Non-responders $(n=73)$

Call Outcomes: we were unable to contact $38 \%$ of non-responders, $46 \%$ was due to an incorrect or no phone number. Of those contacted main reasons for non-participation were: 'not receiving a kit' $(n=19)$ and 'I do not want to be screened' $(n=14) .66 \%(n=28)$ of patients contacted agreed to screening. Of these 28 patients $50 \%$ completed screening, all with a negative result. 15 patients refused screening following our calls, 14 of these stating 'they didn't want to know if they had cancer'.

Caller: Protected time was allocated for the doctor but not the HCA therefore more calls were made by the doctor ( $\mathrm{Dr} n=46, \mathrm{HCA}$ $\mathrm{n}=27$ ), however looking at call outcomes calls from doctor and HCA had similar rates of screening uptake. From calls made by the doctor $39 \%(n=18)$ of patients agreed to screening and of these $44 \%(n=8)$ completed screening. From calls made by HCA $33 \%$ $(n=9)$ agreed to screening and of these $66 \%(n=6)$ completed screening. One patient agreed to screening following an opportunistic discussion within a consultation.

Ethnicity: $65 \%$ of non-responders were British and spoke English as their first language, 12 of the 15 people who refused screening following our calls were British.

Rising 60's $(n=26)$ 
BMJ Quality Improvement Reports

Within the rising 60 's we had a screening uptake of $46 \%$. This appears to be an increase from our previous uptake of $32.72 \%$ however, this is only looking at 'rising 60's' and does not include patients eligible for two yearly re-screening, so we cannot fully calculate our actual increase.

Opportunistic Promotion

The project and results were presented at our practice meeting and written feedback was obtained from six doctors. All doctors were aware that the LES was running and five of the six had received an information pack and sample screening kit. All five reported that this was useful and four of the five had used it within the consultation as a demonstration. All six felt that the alerts of the screen had been helpful and all six had managed to discuss screening within the consultation ranging from one to twenty times. The main barrier to discussion was time, the doctors felt they had too much to cover already in 10 minutes and fitting in another discussion was difficult.

\section{Lessons and limitations}

When starting this project we wondered if the high ethnic diversity of our practice was a barrier to screening. In fact the majority of nonresponders were British suggesting perhaps within our practice this is as large a barrier as we suspected. However this is just a 'snap shot' view and to draw formal conclusions we would need to consider how this relates proportionally to our practice ethnic breakdown and look at larger numbers.

Almost all patients who refused participation said 'they did not want to know'. Perhaps this suggests that more so than language and ethnicity a larger barrier may be attitude toward, and understanding of, early detection of malignancy. It would appear that there is a place for increased education amongst our patients regarding this.

The majority of people had a positive response to our calls suggesting there is benefit to active encouragement. The difficulty we had contacting patients was an unexpected and concerning outcome and measures will be implemented to address this.

The similar responses to both callers (Doctor and HCA) seem to suggest that perhaps 'having the conversation' is more important than 'who is having it'. This has helped us plan the sustainability of the project and, whilst it will still be important for clinicians to be aware of screening and promote and discuss this when possible, our HCA will lead the project and continue the phone calls herself therefore freeing up doctor time.

\section{Conclusion}

Overall our interventions improved screening uptake at the practice and will be continued in future. Engaging patients in screening has many challenges but we have identified some barriers within our practice that we can overcome and have implemented a sustainable system which should hopefully continue to increase our screening uptake.

\section{References}

1. Camden Clinical Commissioning Group, Bowel Cancer Screening in Camden: Locally Enhanced Service. 2012/13

2. National General Practice Profiles. Accessed April 2014; Available from: http://fingertips.phe.org.uk/profile/general-practice/data.

3. National Cancer Intelligence Network, GP Profiles for Cancer. 2013; Available from: http://www.ncin.org.uk/cancer_information_tools/profiles/gp profiles

4. English Indices of Deprivation. 2010, http://www.gov.uk/government/publications/english-indicesof-deprivation-2010,

5. Von Wagner, C., Baio, G., Raine R., Snowball, J., Morris, S., Atkin, W., et al., Inequalities in participation in an organized national colorectal cancer screening programme: results from the first 2.6 million invitations in England, International Journal of Epidemiology, 2011. 40: p. 712-718

6. Cancer Research UK, Bowel Cancer Screening The Facts. 2012, Available from: http://www.cancerresearchuk.org

7. Hewitson P, Glasziou PP, Irwig L, Towler B, Watson E., Screening for colorectal cancer using the faecal occult blood test; Hemoccult (Review), The Cochrane Database of Systematic Reviews (1) 2007

\section{Declaration of interests}

nothing to declare

\section{Acknowledgements}

I would like to acknowledge team members Zuzanna Kwiatkowska and Lyndsey Roome and my trainer Dr Richard Walthew. I would also like to acknowledge the London Deanery Population Health Programme particularly Andrew Burnett, Tara Gray, Debra de Silva and Fiona Simm. 